\title{
ON SUBGRAPHS OF THE \\ COMPLETE BIPARTITE GRAPH
}

P. Erdôs and J. W. Moon

(received March 22, 1963)

$G(n)$ denotes a graph of $n$ vertices and $\bar{G}(n)$ denotes its complementary graph. In a complete graph every two distinct vertices are joined by an edge. Let $C_{k}(G(n))$ denote the number of complete subgraphs of $k$ vertices contained in $G(n)$. Recently it was proved [1] that for every $k$

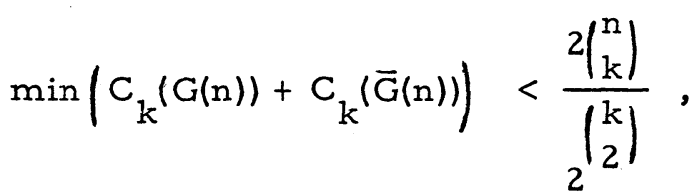

where the minimum is over all graphs $G(n)$. It seems likely that (1) is not far from being best possible and that

$$
\lim _{n \rightarrow \infty} \min \frac{C_{k}(G(n))+C_{k}(\bar{G}(n))}{\left(\begin{array}{l}
n \\
k
\end{array}\right)}=\frac{2}{\left(\begin{array}{l}
k \\
2
\end{array}\right)} .
$$

That this is true for $k=3$ follows from the results of Goodman [2], Sauvé [5], and Lorden [3]. We are unable to prove (2) for $k>3$ but we can prove an analogous result for bipartite graphs.

The bipartite graph $B(m, n)$ consists of the vertices $x_{1}, \ldots, x_{m}$ and $y_{1}, \ldots, y_{n}$ and some of the edges $\left(x_{i}, y_{j}\right)$. $\bar{B}(m, n)$ has the same vertices, and the edge $\left(x_{i}, y_{j}\right)$ is in $\bar{B}(m, n)$ if it is not in $B(m, n)$. If $B(m, n)$ contains $m n$

Canad. Math. Bull. vol. 7, no. 1, January 1964 
edges then it will be referred to as the complete $(m, n)$ graph. Let $C_{k, l}(B(m, n))$ denote the number of complete $(k, l)$ graphs contained in $B(m, n)$. We shall prove the following

THEOREM. For fixed positive integers $k$ and $l$

$$
\lim _{\substack{m \rightarrow \infty \\
n \rightarrow \infty}} \min \frac{C_{k, l}(B(m, n))+C_{k, l}(\bar{B}(m, n))}{\left(\begin{array}{l}
m \\
k
\end{array}\right) \cdot\left(\begin{array}{l}
n \\
l
\end{array}\right)}=\frac{2}{2^{k \ell}}
$$

where the minimum is to be taken over all graphs $B(m, n)$ as $\mathrm{m}$ and $\mathrm{n}$ tend to infinity independently.

We shall prove this theorem in two steps. First we observe that

$$
\min \left(C_{k, l}(B(m, n))+C_{k, l}(\bar{B}(m, n))\right)<\frac{2\left(\begin{array}{c}
m \\
k
\end{array}\right)\left(\begin{array}{l}
n \\
l
\end{array}\right)}{2^{k l}} .
$$

The proof of this is quite similar to that of (1) but for the sake of completeness we shall outline it.

There are $\left(\begin{array}{l}m \\ k\end{array}\right)\left(\begin{array}{l}n \\ l\end{array}\right)$ complete $(k, l)$ graphs contained in the complete $(m, n)$ graph. The probability that any one of these is entirely contained in either $B(m, n)$ or $\bar{B}(m, n)$ is clearly $2 / 2^{\mathrm{kl}}$, assuming all possibilities equally likely. Hence, the expected value of $\mathrm{Y}=\mathrm{C}_{k \ell}(B(m, n))+C_{k, l}(\bar{B}(m, n))$ equals the right-hand side of (3). Since $Y$ is greater than the right-hand side when $B(m, n)$ is the complete $(m, n)$ graph, the strict inequality ( 3 ) now follows immediately.

The rest of our proof makes use of the following simple

LEMMA. Let there be given integrable functions $f_{i}(x)$ and $g_{i}(x), i=1,2, \ldots, m$, such that $f_{i}(x)+g_{i}(x)=1$ and $0 \leq f_{i}(x) \leq 1$ for all $0 \leq x \leq 1$. Let 


$$
T=\int_{0}^{1}\left(\Sigma f_{i_{1}}(x) \ldots f_{i_{k}}(x)+\Sigma g_{i_{1}}(x) \ldots g_{i_{k}}(x)\right) d x,
$$

where the sums are over the $\left(\begin{array}{l}m \\ k\end{array}\right)$ unordered k-tuples of distinct functions. Then, for every $\epsilon_{1}>0$,

$$
T \geq\left(1-\epsilon_{1}\right) \frac{2}{2^{k}}\left(\begin{array}{c}
m \\
k
\end{array}\right), \quad \text { if } m>m_{0} \text {. }
$$

\section{Proof. Let}

$$
H=\frac{1}{k !} \int_{0}^{1}\left[\left(\sum_{i=1}^{m} f_{i}(x)\right)^{k}+\left(\sum_{i=1}^{n} g_{i}(x)\right)^{k}\right] d x .
$$

Using the Cauchy-Schwarz inequality and crude estimates, we find that

$$
\frac{2}{k !}\left(\frac{m}{2}\right)^{k} \leq H \leq T+\frac{2}{k !}\left(m^{k}-m_{(k)}\right) .
$$

But $\mathrm{m}^{\mathrm{k}}-\mathrm{m}_{(\mathrm{k})}=0\left(\mathrm{~m}^{\mathrm{k}-1}\right)$, for fixed $\mathrm{k}$, as $\mathrm{m} \rightarrow \infty$. The lemma now follows directly.

We now show that for every $\epsilon>0$

(4) $\quad C_{k, l}(B(m, n))+C_{k, l}(\bar{B}(m, n)) \geq(1-\epsilon) \frac{2}{2^{k l}}\left(\begin{array}{l}m \\ k\end{array}\right)\left(\begin{array}{l}n \\ l\end{array}\right)$, if $\mathrm{m}>\mathrm{m}_{0}$ and $\mathrm{n}>\mathrm{n}_{\mathrm{o}}$

If $\frac{j-1}{n}<x \leq \frac{j}{n}$ set $f_{i}(x)=1$ or 0 according as the edge $\left(x_{i}, y_{j}\right)$ is in $B(m, n)$ or $\bar{B}(m, n)$, respectively, for $i=1,2, \ldots, m$ and $j=1,2, \ldots, n$. Set $f_{i}(0)=0$ and $g_{i}(x)=1-f_{i}(x)$ for all $i$. Label the k-tuples of the $x$ points from 1 to $\left(\begin{array}{c}m \\ k\end{array}\right)^{i}$. Let $t_{v}$ and $h_{v}$ denote the number of points $y$ for which the edge 
$\left(x_{i}, y\right)$ is in $B(m, n)$ or $\bar{B}(m, n)$, respectively, for each point $x_{i}$ in the $v^{\text {th }} k$-tuple of points. It is not difficult to see that

$$
\sum_{v=1}^{\left(\begin{array}{c}
m \\
k
\end{array}\right)}\left(t_{v}+h_{v}\right)=n T .
$$

Applying Jensen's inequality, we have that

$$
\begin{aligned}
& C_{k, l}(B(m, n))+C_{k, l}(\bar{B}(m, n))=\sum_{\nu=1}\left\{\left(\begin{array}{c}
m_{k} \\
v \\
\ell
\end{array}\right)+\left(\begin{array}{l}
h_{v} \\
\ell
\end{array}\right)\right\} \\
& \geq 2\left(\begin{array}{c}
m \\
k
\end{array}\right)\left(\begin{array}{c}
n T / 2\left(\begin{array}{c}
m \\
k
\end{array}\right) \\
\ell
\end{array}\right) .
\end{aligned}
$$

Using the Iemma, a simple calculation shows that this last quantity is greater than or equal to

$$
(1-\epsilon) \frac{2}{2^{k l}}\left(\begin{array}{c}
m \\
k
\end{array}\right)\left(\begin{array}{l}
n \\
l
\end{array}\right) \text {, }
$$

for sufficiently large $m$ and $n$. This completes the proof of (4) and the theorem now follows by combining (3) and (4).

It seems very unlikely that one can replace (4) by an exact lower bound in general. Lower bounds are given in [4] for the case that $m=n$ and $k=l=2$ but it isn' $t$ known if even the se are exact when $n \geq 10$.

In closing, we remark that the above theorem can easily be extended to the case where the edges of the complete $(m, n)$ graph are split into an arbitrary number of classes instead of just two, as supposed here. 


\section{REFERENCES}

1. P. Erdös, On the number of complete subgraphs contained in certain graphs, Publ. Math. Inst. Hung. Acad. Sci. 7 (1962), 459-464.

2. A. W. Goodman, On sets of acquaintances and strangers at any party, Amer. Math. Monthly, 66 (1959) 778-783.

3. G. Lorden, Blue-empty chromatic graphs, Amer. Math. Monthly, 69 (1962) 114-120.

4. J. W. Moon and L. Moser, On chromatic bipartite graphs, Math. Mag. 35 (1962) 225-227.

5. L. Sauvé, On chromatic graphs, Amer. Math. Monthly, 68 (1961) 107-111.

University College, London 